\title{
Critical evaluation of ceftolozane-tazobactam for complicated urinary tract and intra-abdominal infections
}

This article was published in the following Dove Press journal:

Therapeutics and Clinical Risk Management

19 May 2016

Number of times this article has been viewed

\author{
Stephanie E Giancola' \\ Monica V Mahoney ${ }^{2}$ \\ Tiffany E Bias ${ }^{3,4}$ \\ Elizabeth B Hirsch ${ }^{2,5}$ \\ 'Department of Pharmacy, St Mary's \\ Medical Center, Huntington, WV, ${ }^{2}$ Beth \\ Israel Deaconess Medical Center, \\ Boston, MA, ${ }^{3}$ Hahnemann University \\ Hospital, ${ }^{4}$ Drexel University College \\ of Medicine, Philadelphia, PA, \\ ${ }^{5}$ Department of Pharmacy and Health \\ Systems Sciences, Northeastern \\ University, Boston, MA, USA
}

\begin{abstract}
The rise in resistant Gram-negative pathogens continues to challenge clinicians treating infections. These resistant infections have inspired the development of new antimicrobial agents, including ceftolozane-tazobactam, a novel $\beta$-lactam/ $\beta$-lactamase inhibitor combination approved by the US Food and Drug Administration for the treatment of complicated urinary tract infections (cUTIs) and complicated intra-abdominal infections (cIAIs) in combination with metronidazole. Ceftolozane exhibits bactericidal activity by inhibiting penicillin-binding proteins (PBPs), with high affinity for PBP1b, PBP1c, and PBP3. The addition of tazobactam protects ceftolozane from hydrolysis by irreversibly binding to some $\beta$-lactamase enzymes. Ceftolozane-tazobactam is active against a wide range of Gram-negative pathogens, including extended-spectrum $\beta$-lactamase (ESBL)-producing Enterobacteriaceae and multidrug-resistant (MDR) Pseudomonas aeruginosa, several streptococcal species, and Bacteroides fragilis. When anaerobic coverage is needed, it should be used in combination with metronidazole. Ceftolozane demonstrates linear pharmacokinetics, low protein binding, and minimal accumulation with repeated dosing. The major pharmacokinetic/pharmacodynamic index for ceftolozane is the percentage of the dosing interval in which the plasma free drug concentration remains higher than the minimum inhibitory concentration ( $\% \mathrm{~T}>\mathrm{MIC})$. Phase III clinical trials for the treatment of cUTIs and cIAIs have been completed, showing that it is an effective and safe alternative for the treatment of these infections. The approved dose for cUTIs and cIAIs is $1.5 \mathrm{~g}$ ( $1 \mathrm{~g}$ ceftolozane and $500 \mathrm{mg}$ tazobactam) infused over 1 hour every 8 hours. A higher $3 \mathrm{~g}$ dose is currently in Phase III trials for the treatment of ventilated nosocomial pneumonia. Dosage adjustments are necessary for patients with moderate-to-severe renal impairment. Current data suggest that ceftolozane-tazobactam is a promising carbapenem-sparing alternative agent for the treatment of cUTIs and cIAIs, including those caused by ESBL-producing Enterobacteriaceae and MDR P. aeruginosa.
\end{abstract}

Keywords: multidrug-resistant, UTI, resistance, $\beta$-lactamase

\section{Introduction to the management issues in the treatment of complicated urinary tract infections and complicated intra-abdominal infections}

The threat of antimicrobial resistance among bacterial infections is widespread and continues to increase, especially among Gram-negative pathogens. ${ }^{1,2}$ Of particular concern are organisms of the Enterobacteriaceae family, as well as Pseudomonas aeruginosa. Extended-spectrum $\beta$-lactamase (ESBL)-producing Enterobacteriaceae, carbapenem-resistant Enterobacteriaceae (CRE), and multidrug-resistant (MDR) $P$. aeruginosa account for $\sim 26,000, \sim 9,000$, and $\sim 6,700$ health care-associated infections, respectively, in the USA each year. ${ }^{2}$ Complicated urinary tract infections
Correspondence: Elizabeth B Hirsch Systems Sciences, Northeastern University, 360 Huntington Avenue, R2 I 8 TF, Boston, MA 02II5, USA

Tel +l 6173737973

Fax + I 6II 3737655

Email e.hirsch@neu.edu
Therapeutics and Clinical Risk Management 2016:12 787-797

787

Dovepress

http://dx.doi.org/10.2147/TCRM.S83844

(c) (1) () 2016 Giancola et al. This work is published and licensed by Dove Medical Press Limited. The full terms of this license are available at https://www.dovepress.com/terms.php (c)
hereby accept the Terms. Non-commercial uses of the work are permitted without any further permission from Dove Medical Press Limited, provided the work is properly attributed. For permission

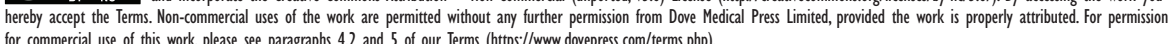


(cUTIs) and complicated intra-abdominal infections (cIAIs) are especially affected by the increase in resistant Gramnegative pathogens. ${ }^{3,4}$

cUTIs occur in the presence of structural or functional urinary tract abnormalities, such as urolithiasis, indwelling catheters, neurogenic bladder, or increased postvoid residual volume, and are associated with a higher rate of recurrence, reinfection, and resistant pathogens. ${ }^{5,6}$ While a broader range of pathogens, including Klebsiella spp., P. aeruginosa, and Enterococcus spp., cause cUTIs compared with uncomplicated infection, Escherichia coli remains the most common., Longer courses of intravenous (IV) or oral antibiotics, including fluoroquinolones, $\beta$-lactams, and aminoglycosides, are typically used to treat cUTIs. ${ }^{7}$ However, treatment decisions for UTIs have become more difficult in the face of increased Gram-negative resistance. Zilberberg and Shorr ${ }^{3}$ examined The Surveillance Network Database to estimate the prevalence of drug resistance among uropathogens isolated from hospitalized patients in the USA. The study demonstrated an increase from $3.3 \%$ to $8 \%$ in ESBL-producing E. coli, $9.1 \%$ to $18.6 \%$ in ESBL-producing $K$. pneumoniae, and $0 \%$ to $2.3 \%$ in CRE causing UTIs in the period 2000-2009. ${ }^{3}$ Additionally, among catheter-associated UTIs reported to the National Healthcare Safety Network ${ }^{1}$ in 2009-2010, $12.3 \%$ and $2.3 \%$ of $E$. coli isolates, $26.9 \%$ and $12.5 \%$ of Klebsiella isolates, and $25.2 \%$ and $21.3 \%$ of $P$. aeruginosa isolates were resistant to extended-spectrum cephalosporins and carbapenems, respectively.

Complicated IAIs extend into the peritoneal space as a result of perforation of the gastrointestinal tract and lead to abscess formation or peritonitis. ${ }^{8}$ These infections are often polymicrobial and involve facultative and aerobic Gram-negative organisms, Gram-positive aerobes, and anaerobic bacteria. The most common pathogens causing cIAIs include Enterobacteriaceae (especially E. coli),
Bacteroides spp., and Streptococcus spp. Health careassociated infections are often caused by drug-resistant pathogens, such as ESBL-producing Enterobacteriaceae and MDR $P$. aeruginosa.$^{8,9}$ Babinchak et $\mathrm{al}^{4}$ reported an increase from $1.7 \%$ to $7.3 \%$ in ESBL-producing $E$. coli and $3.2 \%$ to $13.1 \%$ in ESBL-producing $K$. pneumoniae causing IAIs in patients from 37 hospitals in the USA and Canada between 2005 and 2010. The management of cIAIs includes antimicrobial therapy in combination with an appropriate source control procedure, such as surgery or percutaneous abscess drainage. Empiric antimicrobial therapy generally includes coverage of a wide variety of pathogens with the use of a cephalosporin or fluoroquinolone plus metronidazole, a carbapenem, or piperacillin-tazobactam. ${ }^{8}$

The continuous rise in Gram-negative bacterial resistance to the available antimicrobial agents has inspired the development of new agents to treat these resistant infections. ${ }^{7,9}$ This review focuses on ceftolozane-tazobactam, a $\beta$-lactam $/ \beta$ lactamase inhibitor combination for cUTIs and cIAIs, recently approved by the US Food and Drug Administration (FDA).

\section{Current treatment options for managing MDR Gram-negative bacterial infections}

Gram-negative bacterial resistance to $\beta$-lactam antibiotics most commonly occurs due to the production of $\beta$-lactamase enzymes that hydrolyze the $\beta$-lactam ring, resulting in decreased antibiotic activity. ${ }^{10,11}$ More than 1,400 $\beta$-lactamase enzymes have been described; when using the Ambler classification system, they are divided into four classes, as depicted in Table $1 .{ }^{10}$ Ambler classes A, C, and D enzymes act through a serine intermediate, while class B enzymes, often referred to as metallo- $\beta$-lactamases, use zinc to hydrolyze the $\beta$-lactam ring. ${ }^{11,12}$ Class A enzymes include narrow-spectrum

Table I Ambler classification of $\beta$-lactamases with examples

\begin{tabular}{|c|c|c|c|c|}
\hline Class & Active site & Enzyme type & Common organisms & Examples \\
\hline A & Serine & Narrow spectrum & Escherichia coli, Klebsiella pneumoniae & $\begin{array}{l}\text { Staphylococcal penicillinase, TEM-I, } \\
\text { TEM-2, SHV-I }\end{array}$ \\
\hline A & Serine & Extended spectrum & Enterobacteriaceae, Pseudomonas aeruginosa & SHV-derived, CTX-M, PER-I, VEB-I \\
\hline A & Serine & Carbapenemases & K. pneumoniae & KPC-derived, IMI-I \\
\hline B & Zinc & $\begin{array}{l}\text { Metallo- } \beta \text {-lactamases } \\
\text { (carbapenemases) }\end{array}$ & $\begin{array}{l}\text { Bacteroides fragilis, Stenotrophomonas maltophilia, } \\
\text { P. aeruginosa, Acinetobacter baumannii }\end{array}$ & IMP-I, VIM-I, NDM-I \\
\hline C & Serine & Cephalosporinases & $\begin{array}{l}\text { Enterobacter spp., Citrobacter spp., Proteus spp., } \\
\text { Serratia spp., P. aeruginosa }\end{array}$ & $\begin{array}{l}\text { AmpC, P99, ACT-I, CMY-2, MIR-I, } \\
\text { FOX-I }\end{array}$ \\
\hline D & Serine & $\begin{array}{l}\text { OXA-type enzymes } \\
\text { (ESBLs, carbapenemases) }\end{array}$ & A. baumannii, $P$. aeruginosa, E. coli & OXA-derived \\
\hline
\end{tabular}

Notes: Data from Toussaint and Gallagher; ${ }^{10}$ Bush and Jacoby; ${ }^{\prime \prime}$ Liscio et al; ${ }^{12}$ and Kaye and Pogue. ${ }^{16}$

Abbreviation: ESBL, extended-spectrum $\beta$-lactamase. 
$\beta$-lactamases, ESBLs, and carbapenemases. ${ }^{10,12}$ Except for the carbapenemases, these enzymes are generally inhibited in vitro by the $\beta$-lactamase inhibitors clavulanic acid, sulbactam, and tazobactam. Avibactam, a novel $\beta$-lactamase inhibitor, has shown promising activity against class A carbapenemases. ${ }^{10}$ Class B enzymes are generally carbapenemases and are not inhibited by the available $\beta$-lactamase inhibitors. Class $\mathrm{C}$ enzymes are often chromosomally mediated, inducible cephalosporinases such as AmpC, which are usually produced by the organisms in the "SPACE" genera (Serratia, Pseudomonas, Acinetobacter, Citrobacter, and Enterobacter). Class D enzymes are usually ESBLs and carbapenemases. ${ }^{10,12}$

Additional resistance mechanisms among $P$. aeruginosa strains include loss of the OprD porin and overproduction of efflux pumps. ${ }^{13,14}$ Loss of the OprD porin causes decreased permeability of the outer membrane, conferring resistance to carbapenems. The overproduction of efflux pumps by $P$. aeruginosa leads to resistance to fluoroquinolones and some $\beta$-lactam antibiotics. ${ }^{15}$

Carbapenem antibiotics have long been considered the drugs of choice for infections caused by ESBL-producing organisms. ${ }^{10,16}$ Mounting clinical outcomes data have demonstrated that cefepime and piperacillin-tazobactam may be reasonable carbapenem-sparing alternatives against ESBL-producing E. coli and Klebsiella spp. in less serious infections, or when the minimum inhibitory concentrations (MICs) are $\leq 2 \mu \mathrm{g} / \mathrm{mL}$ for cefepime and $\leq 16 \mu \mathrm{g} / \mathrm{mL}$ for piperacillin-tazobactam. ${ }^{17-19}$

The treatment of CRE is particularly problematic as data are based primarily on small, single-center, retrospective studies. ${ }^{16}$ Combination therapy is generally preferred and most of the regimens commonly involve polymyxins, tigecycline, aminoglycosides, and high-dose, prolonged-infusion carbapenems. ${ }^{16,20-22}$ The recently FDA-approved $\beta$-lactam/ $\beta$ lactamase inhibitor combination ceftazidime-avibactam has demonstrated in vitro activity against CRE and may represent a possible treatment option. ${ }^{12}$ It is currently in Phase III trials for the treatment of cUTIs (NCT01595438, NCT01599806), cIAIs, (NCT01500239, NCT01499290, NCT01726023) and nosocomial pneumonia (NCT01808092). ${ }^{23}$

Treatment of MDR $P$. aeruginosa usually involves sufficiently high doses of active antipseudomonal $\beta$-lactam antibiotics, including cefepime, ceftazidime, piperacillintazobactam, aztreonam, or a carbapenem. ${ }^{16}$ Some clinicians may treat with combination therapy by adding an aminoglycoside or antipseudomonal fluoroquinolone, especially for empiric treatment. Polymyxins, either as monotherapy or as combination therapy, are generally used as salvage therapy when no other agents with in vitro activity remain. ${ }^{24,25}$

Ceftolozane-tazobactam, the focus of the remainder of this review, represents a carbapenem-sparing treatment option for ESBL-producing organisms and MDR P. aeruginosa, as described in later sections.

\section{Review of pharmacology, mode of action, microbiology, and pharmacokinetics of ceftolozane- tazobactam}

\section{Structure and mechanism of action}

Ceftolozane-tazobactam is a new $\beta$-lactam/ $\beta$-lactamase inhibitor combination approved by the FDA for the treatment of cUTIs, as well as for cIAIs in combination with metronidazole. ${ }^{26}$ Ceftolozane is a novel oxyimino-aminothiazolyl cephalosporin similar in structure to ceftazidime. Its bactericidal activity is a result of the inhibition of bacterial cell wall synthesis mediated by binding to penicillin-binding proteins (PBPs). Ceftolozane inhibits the PBPs important in $P$. aeruginosa (PBP1b, PBP1c, and PBP3) as well as in E. coli (PBP3) with a greater affinity than ceftazidime. ${ }^{26,27}$ Ceftolozane possesses enhanced activity against Gramnegative pathogens, including $P$. aeruginosa and Enterobacteriaceae. Ceftolozane appears to be less susceptible to hydrolysis by AmpC $\beta$-lactamases than other cephalosporins due to decreased affinity to PBP4 and also has activity against bacteria with efflux pumps and porin deficiencies due to its increased binding to PBP3. ${ }^{28-30}$ Similar to other cephalosporins, it is hydrolyzed by ESBLs; therefore, it was developed in combination with tazobactam in order to broaden its antimicrobial activity. ${ }^{31}$ Tazobactam is an established $\beta$-lactamase inhibitor that protects ceftolozane from hydrolysis by irreversibly binding to some $\beta$-lactamase enzymes. ${ }^{10}$ Tazobactam inhibits most class A narrowspectrum $\beta$-lactamases and ESBLs, as well as some class $\mathrm{C}$ enzymes, thus enhancing ceftolozane's activity against ESBL-producing bacteria and some anaerobes. It does not inhibit most class B or class D enzymes. By itself, tazobactam does not have clinically significant in vitro activity against bacteria. ${ }^{10,27}$

\section{Microbiology}

The combination of ceftolozane and tazobactam is active against a wide range of Gram-negative pathogens, including ESBL-producing organisms and MDR $P$. aeruginosa, many streptococcal species, and Bacteroides fragilis. It is still 
recommended, however, to be used in combination with metronidazole for anaerobic coverage as described in the following section. It has limited activity against Staphylococcus spp. and other anaerobes. ${ }^{12,26,27}$ The FDA breakpoints are listed in Table 2. Clinical and Laboratory Standards Institute ${ }^{32}$ breakpoints have not yet been established.

Several studies have evaluated the in vitro activity of ceftolozane-tazobactam against clinical isolates. ${ }^{31,33,34}$ Farrell et $\mathrm{a}^{33}$ tested the susceptibility of 7,071 Enterobacteriaceae and 1,971 $P$. aeruginosa isolates from hospitalized patients in the USA with documented infections. Ceftolozane-tazobactam was potent against the Enterobacteriaceae overall and remained active against the $601 \mathrm{MDR}$ isolates $\left(\mathrm{MIC}_{50 / 90}=4 />32 \mu \mathrm{g} / \mathrm{mL}\right)$. Meropenem, tigecycline, and colistin also retained activity. Tigecycline was the most active agent against extensively drug-resistant (XDR) strains (87.1\% susceptible), followed by meropenem (22.1\% susceptible) and gentamicin $(20.9 \%$ susceptible); ceftolozane-tazobactam was not active against most XDR strains $\left(\mathrm{MIC}_{50 / 90}=>32 />32 \mu \mathrm{g} / \mathrm{mL}\right)$. Ceftolozanetazobactam demonstrated high potency against $E$. coli $\left(\mathrm{MIC}_{50 / 90}=0.25 / 0.5 \mu \mathrm{g} / \mathrm{mL}\right)$, including the 327 ESBL-producing isolates $\left(\mathrm{MIC}_{50 / 90}=0.5 / 4 \mu \mathrm{g} / \mathrm{mL}\right)$. It was also highly active against non-ESBL $K$. pneumoniae; however, it demonstrated lower activity against ESBL-producing isolates $\left(\mathrm{MIC}_{50 / 90}=32 />32 \mu \mathrm{g} / \mathrm{mL}\right)$. This was explained by the higher rate of carbapenemases produced by $K$. pneumoniae isolates. Ceftolozane-tazobactam was the most potent agent tested against all $P$. aeruginosa isolates $\left(\mathrm{MIC}_{50 / 90}=0.5 / 2 \mu \mathrm{g} /\right.$ $\mathrm{mL}$ ) and second only to colistin against the $310 \mathrm{MDR}$ strains $\left(\mathrm{MIC}_{50 / 90}=2 / 8 \mu \mathrm{g} / \mathrm{mL}\right)$ as well as XDR strains $\left(\mathrm{MIC}_{50 / 90}=4 / 16 \mu \mathrm{g} / \mathrm{mL}\right)$.

In a similar study, ${ }^{31}$ in vitro susceptibility to ceftolozanetazobactam was tested using 1,301 isolates of Enterobacteriaceae, $P$. aeruginos $a$, and $B$. fragilis with varying antimicrobial resistance patterns from patients in the USA, Europe, Latin America, and Asia. Ceftolozane-tazobactam

Table 2 US Food and Drug Administration susceptibility interpretive criteria for ceftolozane-tazobactam

\begin{tabular}{llll}
\hline Pathogen & \multicolumn{3}{l}{ Minimum inhibitory concentration $(\mu \mathrm{g} / \mathrm{mL})$} \\
\cline { 2 - 4 } & Susceptible & Intermediate & Resistant \\
\hline Enterobacteriaceae & $\leq 2 / 4$ & $4 / 4$ & $\geq 8 / 4$ \\
Pseudomonas aeruginosa & $\leq 4 / 4$ & $8 / 4$ & $\geq 16 / 4$ \\
Streptococcal species $^{\mathrm{a}}$ & $\leq 8 / 4$ & $16 / 4$ & $\geq 32 / 4$ \\
Bacteroides fragilis & $\leq 8 / 4$ & $16 / 4$ & $\geq 32 / 4$ \\
\hline
\end{tabular}

Notes: aStreptococcus anginosus, Streptococcus constellatus, Streptococcus salivarius. ZERBAXA product information reproduced with permission of Merck Sharp \& Dohme Corp., a subsidiary of Merck \& Co., Inc., Kenilworth, New Jersey, U.S.A. All rights reserved. ${ }^{26}$ demonstrated good activity against ceftazidime-resistant E. coli $\left(\mathrm{MIC}_{50 / 90}=1 / 16 \mu \mathrm{g} / \mathrm{mL}\right)$ and $K$. pneumoniae isolates $\left(\mathrm{MIC}_{50 / 90}=4 />16 \mu \mathrm{g} / \mathrm{mL}\right)$. Poor activity against the 53 isolates of $K$. pneumoniae that produced carbapenemases was demonstrated $\left(\mathrm{MIC}_{50 / 90}=>16 />16 \mu \mathrm{g} / \mathrm{mL}\right)$. Forty-one $B$. fragilis wild-type strains were tested and ceftolozane-tazobactam showed good activity against these $\left(\mathrm{MIC}_{50 / 90}=1 />16 \mu \mathrm{g} / \mathrm{mL}\right)$. Ceftolozane-tazobactam also showed excellent activity against all $P$. aeruginosa isolates tested $\left(\mathrm{MIC}_{50 / 90}=1 / 8 \mu \mathrm{g} / \mathrm{mL}\right.$ ), including ceftazidime-resistant and/or imipenem-resistant isolates. A study by Snydman et $a^{34}$ evaluated the in vitro activity of ceftolozane-tazobactam against anaerobic bacteria with a focus on B. fragilis. Among the 244 B. fragilis isolates, the addition of tazobactam significantly improved the isolates' susceptibilities, lowering the $\mathrm{MIC}_{50 / 90}$ from $64 / \geq 256 \mu \mathrm{g} / \mathrm{mL}$ to $1 / 4 \mu \mathrm{g} / \mathrm{mL}$. Ceftolozane-tazobactam demonstrated good activity against Prevotella spp., Fusobacterium spp., and Propionibacterium spp. and limited activity against Clostridium spp. and other Bacteroides spp. The authors suggested that while ceftolozane-tazobactam may have clinically significant activity against $B$. fragilis, it should still be used in combination with metronidazole, a more potent antianaerobic agent, in the treatment of cIAIs as the $\mathrm{MIC}_{90}$ of $4 \mu \mathrm{g} / \mathrm{mL}$ was just one dilution below the susceptibility breakpoint of $8 \mu \mathrm{g} / \mathrm{mL}$ for $B$. fragilis.

\section{Pharmacokinetics}

The pharmacokinetic (PK) profile of ceftolozane following single- and multiple-IV infusions over 1 hour was evaluated in a Phase I study that included 64 healthy adult volunteers. ${ }^{35}$ Dose-linear PK profiles were observed across the range of doses studied. Ceftolozane demonstrated an average half-life of 2.3 hours, which was not affected by dose or duration. The change in area under the concentration-time curve (AUC) of ceftolozane was minimal between day 1 and day 10, suggesting minimal accumulation of ceftolozane with repeated dosing. The clearance of ceftolozane was also independent of dose and duration and correlated well with creatinine clearance $\left(\mathrm{CL}_{\mathrm{CR}}\right)$. Approximately $95 \%$ of ceftolozane was excreted unchanged in the urine following repeated doses, suggesting that dosing adjustments are required in renal impairment. Additionally, ceftolozane displayed low protein binding of $\sim 20 \%$ in human plasma.

Another study ${ }^{36}$ examined the PK profile of ceftolozane in 58 healthy adults when given alone or in combination with tazobactam at a 2:1 ratio. Dose-linear PK profiles were observed similar to the previous study and these were comparable whether ceftolozane was given alone or with 
Table 3 Pharmacokinetic values following single- and multiple infusions of ceftolozane-tazobactam $1.5 \mathrm{~g}$ over I hour every 8 hours

\begin{tabular}{|c|c|c|c|c|}
\hline \multirow{2}{*}{$\begin{array}{l}\text { Parameter, } \\
\text { mean (\%CV) }\end{array}$} & \multicolumn{2}{|l|}{ Ceftolozane } & \multicolumn{2}{|l|}{ Tazobactam } \\
\hline & Day I $(n=9)^{a}$ & Day I0 $(n=10)$ & Day I $(n=9)^{a}$ & Day I0 $(n=10)$ \\
\hline$C_{\max }(\mu g / m L)$ & $69.1(11.3)$ & $74.4(13.6)$ & $18.4(15.9)$ & $18.0(8.0)$ \\
\hline$T_{\max }^{\max }$ (hours) $)^{\mathrm{b}}$ & $1.02(1.01-1.1)$ & $1.07(1.0-1.1)$ & $1.02(0.99-1.03)$ & I.0I (I.0-I.I) \\
\hline AUC $(\mu g \cdot h / m L)$ & $172(\mid 3.8)$ & $197(16.6)$ & $24.4(17.9)$ & $24.8(I 5.5)$ \\
\hline Half-life (hours) & $2.77(30)$ & $3.12(21.9)$ & $0.91(26.2)^{c}$ & $1.03(18.6)$ \\
\hline Clearance (L/h) & $5.86(13.7)$ & $5.58(12.6)$ & $20.6(17.8)^{c}$ & 20.4 (I3.6) \\
\hline$V_{s s}(L)$ & $14.6(16.0)$ & $14.2(16.6)$ & $18.1(12.6)^{c}$ & $17.9(9.7)$ \\
\hline
\end{tabular}

Notes: ane patient excluded due to outlying plasma results. ${ }^{b}$ Data presented as median (range). ${ }^{c} n=8$, one patient excluded because concentration-time profile did not exhibit a terminal log-linear phase and therefore half-life, clearance, and $V_{\mathrm{ss}}$ could not be calculated. Antimicrob Agents Chemother, 20I2;56(6):3086-309I, doi: 10.II28/ AAC.06349-II and reproduced with permission from American Society for Microbiology. ${ }^{36}$

Abbreviations: AUC, area under the concentration-time curve; $\mathrm{CV}$, coefficient of variation; $C_{\max }$, maximum plasma concentration; $T_{\max }$, time of maximum plasma concentration; $V_{\mathrm{ss}}$, volume of distribution at steady state.

tazobactam. After repeated dosing, the average elimination half-life of tazobactam and ceftolozane was 1 and 3 hours, respectively. The volume of distribution at steady state was 13.2 and $17.8 \mathrm{~L}$ for ceftolozane and tazobactam, respectively. Ceftolozane was primarily cleared by the kidneys and did not accumulate after repeated dosing. Tazobactam was also primarily eliminated by the kidneys, but metabolism to the inactive M1 metabolite also occurred. Table 3 displays the PK values of ceftolozane-tazobactam at $1.5 \mathrm{~g}$ administered every 8 hours, the approved dose for the treatment of cUTIs and cIAIs in patients with normal renal function.

PK studies of ceftolozane-tazobactam have been carried out in subjects with varying degrees of renal function, including those with end-stage renal disease on hemodialysis. ${ }^{37}$ Subjects with normal renal function $\left(\mathrm{CL}_{\mathrm{CR}}:>90 \mathrm{~mL} /\right.$ min) and mild renal impairment $\left(\mathrm{CL}_{\mathrm{CR}}: 60-90 \mathrm{~mL} / \mathrm{min}\right)$ displayed similar, linear PK profiles. Subjects with moderate $\left(\mathrm{CL}_{\mathrm{CR}}: 30-59 \mathrm{~mL} / \mathrm{min}\right)$ and severe renal impairment
$\left(\mathrm{CL}_{\mathrm{CR}}: 15-29 \mathrm{~mL} / \mathrm{min}\right)$ had increased exposures of ceftolozane and tazobactam. In subjects with moderate renal impairment, the AUC increased 2.5-fold and 2.2-fold for ceftolozane and tazobactam, respectively, compared with those with normal renal function. The AUC was increased in severe renal impairment by 4.4 -fold and 3.8-fold for ceftolozane and tazobactam, respectively. The median half-lives of ceftolozane and tazobactam were significantly prolonged in subjects with end-stage renal disease $\left(\mathrm{CL}_{\mathrm{CR}}:<15 \mathrm{~mL} / \mathrm{min}\right)$, resulting in a substantial increase in the AUC. In subjects receiving hemodialysis, the ceftolozane and tazobactam plasma concentrations rapidly decreased following the start of hemodialysis; however, this was followed by a slow redistribution of ceftolozane-tazobactam after hemodialysis ended. The overall exposures of ceftolozane and tazobactam based on AUCs were reduced by $\sim 66 \%$ and $\sim 56 \%$ following dialysis, respectively. The PK parameters determined by this study are presented in Table 4.

Table 4 Pharmacokinetic values following single-dose administration of ceftolozane-tazobactam in subjects with varying degrees of renal function

\begin{tabular}{|c|c|c|c|c|c|c|}
\hline \multirow{2}{*}{$\begin{array}{l}\text { Parameter, } \\
\text { median } \\
\text { (range) }\end{array}$} & \multirow{2}{*}{$\begin{array}{l}\text { Normal } \\
(\mathrm{n}=\mathrm{II}), \mathrm{I} .5 \mathrm{~g}\end{array}$} & \multirow{2}{*}{$\begin{array}{l}\text { Mild } \\
\text { impairment } \\
(\mathrm{n}=6), 1.5 \mathrm{~g}\end{array}$} & \multirow{2}{*}{$\begin{array}{l}\text { Moderate } \\
\text { impairment } \\
(n=7), 1.5 \mathrm{~g}\end{array}$} & \multirow{2}{*}{$\begin{array}{l}\text { Severe } \\
\text { impairment } \\
(\mathrm{n}=6), 750 \mathrm{mg}\end{array}$} & \multicolumn{2}{|l|}{$\operatorname{ESRD~}(n=6)$} \\
\hline & & & & & $\begin{array}{l}\text { Non-HD (day I), } \\
750 \text { mg }\end{array}$ & $\begin{array}{l}\text { HD (day 4), } \\
750 \mathrm{mg}\end{array}$ \\
\hline \multicolumn{7}{|l|}{ Ceftolozane } \\
\hline$C_{\max }(\mu g / m L)$ & $72.8(42-139)$ & $93.4(75.8-|4|)$ & $84.5(64-136)$ & $47.0(37.5-76.3)$ & $44.2(30.2-60.6)$ & $4 I . I(17.5-56.4)$ \\
\hline AUC $(\mu \mathrm{g} \cdot \mathrm{h} / \mathrm{mL})$ & $23 \mid(|6|-3 \mid I)$ & 315 (255-342) & 589 (306-900) & 509 (429-762) & I,629 (466-2,750) & $574(287-I, 024)$ \\
\hline Half-life (hours) & $3.1(2.4-3.6)$ & $3.3(2.9-3.8)$ & $5.6(2.9-10.8)$ & II.I (7.7-14.9) & $40.5(20.8-58.1)$ & $43.2(32.8-56.9)^{\mathrm{a}}$ \\
\hline Clearance (L/h) & $4.3(3.2-6.2)$ & $3.2(2.9-3.9)$ & $1.7(1.1-3.3)$ & $1.0(0.7-1.2)$ & $0.3(0.2-1.1)$ & $0.9(0.5-1.7)$ \\
\hline $\begin{array}{l}V_{\text {ss }}(\mathrm{L}) \\
\text { Tazobactam }\end{array}$ & $14.6(8.9-24.7)$ & $12.3(9.2-13)$ & $13.9(10.6-18.6)$ & $12.5(\mid 1.3-20.4)$ & $17.9(11.9-31.7)$ & $54.6(38.8-77.9)$ \\
\hline$C_{\max }(\mu g / \mathrm{mL})$ & $\mid 7.0(|4.7-3| .4)$ & $21.9(18.9-28.3)$ & 27.1 (23.3-28.7) & $16.3(10.2-18.3)$ & $20.2(15.9-30.3)$ & I 4.9 (7.2-22.9) \\
\hline $\mathrm{AUC}(\mu \mathrm{g} \cdot \mathrm{h} / \mathrm{mL})$ & $30.1(21.7-40.4)$ & $34.7(29.1-43.4)$ & 65.9 (49.1-91.9) & $56.5(35.8-70.9)$ & $109(46.0-170)$ & $40.3(23.3-58.6)$ \\
\hline Half-life (hours) & I.I $(0.8-1.6)$ & I.I (0.9-I.6) & I.8 (I.4-2.2) & $2.5(1.9-3.3)$ & $4.2(3.4-9.1)$ & $5.0(1.9-8.5)^{\mathrm{a}}$ \\
\hline Clearance (L/h) & $16.6(12.4-23.0)$ & I4.4 (II.5-I7.2) & $7.6(5.4-10.2)$ & $4.4(3.5-7.0)$ & $2.4(1.5-5.4)$ & $6.2(4.3-10.7)$ \\
\hline$V_{s s}(L)$ & $19.9(13.8-26.1)$ & $16.0(\mid 2.7-22.0)$ & $16.8(|3.9-2| . \mid)$ & $15.7(\mid 2.2-23.5)$ & $15.2(\mid 1.5-27.1)$ & $27.4(15.4-56.7)$ \\
\hline
\end{tabular}

Notes: Renal function based on creatinine clearance: normal renal function, $>90 \mathrm{~mL} / \mathrm{min}$; mild impairment, $60-90 \mathrm{~mL} / \mathrm{min}$; $\mathrm{moderate}$ impairment, $30-59 \mathrm{~mL} / \mathrm{min}$; severe impairment, 15-29 mL/min; end-stage renal disease, $<15 \mathrm{~mL} / \mathrm{min}$. ${ }^{\text {TT }}$ The half-life on HD was calculated from the post-HD terminal elimination phase. Antimicrob Agents Chemother, 2014;58(4):2249-2255, doi: 10.1 I28/AAC.02151-13 and reproduced with permission from American Society for Microbiology. ${ }^{37}$

Abbreviations: $A \cup C$, area under the concentration-time curve; $C_{\max }$, maximum plasma concentration; ESRD, end-stage renal disease; HD, hemodialysis; $V_{\mathrm{ss}}$, volume of distribution at steady state. 


\section{Pharmacodynamics}

Similar to other beta-lactams, the major PK/pharmacodynamic (PD) index for ceftolozane is the percentage of the dosing interval in which the plasma free drug concentration remains higher than the MIC $(\% \mathrm{~T}>\mathrm{MIC}) .{ }^{28,29} \mathrm{Neu}-$ tropenic mouse thigh-infection models were used to study the PD activity of ceftolozane against $P$. aeruginosa and Enterobacteriaceae. ${ }^{29}$ In order to determine the primary $\mathrm{PK} / \mathrm{PD}$ index, the investigators correlated the number of bacteria in the thigh at the end of 24 hours of therapy with the maximum plasma concentration/MIC ratio, AUC/MIC ratio, and $\% \mathrm{~T}>\mathrm{MIC}$. The $\% \mathrm{~T}>\mathrm{MIC}$ had the highest $R^{2}$ value at $61 \%$, indicating the best correlation with the colony-forming units (CFUs) per thigh. The \% $>$ MIC required for stasis and $1-\log$ kill ranged from $21.4 \%$ to $28.5 \%$ and $26.7 \%$ to $35.3 \%$, respectively, for $P$. aeruginosa and Enterobacteriaceae. The impact of tazobactam on the activity against ESBL-producing Enterobacteriaceae was also evaluated by comparing ceftolozane alone and in combination with tazobactam at 2:1, 4:1, and 8:1 ratios. Compared to mice treated with ceftolozane alone, only ceftolozane and tazobactam at a 2:1 ratio exhibited a significantly greater decrease in $\log _{10}$ CFUs per thigh $(P=0.048)$, suggesting that this was the most effective formulation studied.

The $\% \mathrm{~T}>\mathrm{MIC}$ targets for the antibacterial effect of ceftolozane-tazobactam against several E. coli and P. aeruginosa strains were determined in an in vitro PK model. ${ }^{38}$ Five $E$. coli strains and five $P$. aeruginosa strains with varying MICs were tested. A clear relationship was observed between antibacterial effect and $\% \mathrm{~T}>\mathrm{MIC}$. The $\% \mathrm{~T}>\mathrm{MIC}$ at 24 hours required for stasis, as well as $1-\log , 2-\log$, and $3-\log$ reductions in $\mathrm{CFU}$, for $E$. coli were $27.8 \%, 33.0 \%, 39.4 \%$, and $44.2 \%$, respectively. There was no difference between nonESBL and ESBL-producing strains. Similarly, the \%T $>$ MIC at 24 hours required for stasis, as well as $1-\log , 2-\log$, and 3 - log reductions in CFUs, for P. aeruginosa were $24.9 \%$, $26.6 \%, 31.2 \%$, and $41.5 \%$, respectively, with no difference observed between strains with differing MICs. These studies indicate that the $\% \mathrm{~T}>\mathrm{MIC}$ target required for static effect is lower than the $30 \%-40 \%$ that is typically associated with other cephalosporins, suggesting that ceftolozane's bactericidal activity is more similar to carbapenems..$^{29,38}$

\section{Dosing}

The significant decrease in clearance of ceftolozanetazobactam in patients with impaired renal function warrants a reduction in dosage in some patients. The dosage recommendations by renal function for the treatment of cIAIs
Table 5 Recommended dosages of ceftolozane-tazobactam by renal function for the treatment of clAls and cUTls

\begin{tabular}{ll}
\hline $\begin{array}{l}\text { Estimated } \\
\mathrm{CL}_{\mathrm{CR}}(\mathbf{m L} / \mathbf{m i n})^{\mathrm{a}}\end{array}$ & Dose $^{\mathrm{b}, \mathrm{c}}$ \\
\hline$>50$ & I.5 g IV every 8 hours \\
$30-50$ & $750 \mathrm{mg}$ IV every 8 hours \\
I5-29 & $375 \mathrm{mg}$ IV every 8 hours \\
ESRD on HD & $750 \mathrm{mg}$ IV loading dose, followed by I50 mg \\
& every 8 hours; administer at earliest time \\
& possible after completion of dialysis \\
\hline
\end{tabular}

Notes: ${ }^{a} \mathrm{CL}_{\mathrm{CR}}$ estimated using Cockgroft-Gault equation. ${ }^{\mathrm{b}} \mathrm{All}$ doses are available as a 2:I ratio of ceftolozane and tazobactam and are expressed as the sum of the two active ingredients (ie, $1.5 \mathrm{~g}$ ceftolozane-tazobactam consists of I $\mathrm{g}$ ceftolozane and $0.5 \mathrm{~g}$ tazobactam). 'A All doses are infused over I hour. ZERBAXA product information reproduced with permission of Merck Sharp \& Dohme Corp., a subsidiary of Merck \& Co., Inc., Kenilworth, New Jersey, U.S.A. All rights reserved. ${ }^{26}$

Abbreviations: cIAl, complicated intra-abdominal infection; $\mathrm{CL}_{\mathrm{CR}}$, creatinine clearance; cUTI, complicated urinary tract infection; ESRD, end-stage renal disease; $H D$, hemodialysis; IV, intravenous.

and cUTIs are listed in Table $5 .{ }^{26}$ All recommended doses consist of a 2:1 ratio of ceftolozane and tazobactam and are presented as the sum of the two active ingredients (ie, $1.5 \mathrm{~g}$ ceftolozane-tazobactam is composed of $1 \mathrm{~g}$ ceftolozane and $0.5 \mathrm{~g}$ tazobactam). The original label approved by the FDA expressed the dose as $1 \mathrm{~g} / 0.5 \mathrm{~g}$ to denote the amount of each ingredient. However, in response to seven reported medication errors that occurred during preparation due to confusion with the presentation of the dose, the FDA issued a warning about the risk for dosing errors..$^{39}$ The labeling was then changed to represent the sum of the active ingredients as is seen with other $\beta$-lactam/ $\beta$-lactamase inhibitors.

\section{Comparative efficacy, safety, and tolerability} Clinical trials

Phase III trials have been completed for ceftolozane-tazobactam in the treatment of cUTIs and cIAIs in combination with metronidazole. ${ }^{7,9}$ Assessment of the Safety Profile and Efficacy of Ceftolozane-Tazobactam (ASPECT)-cIAI ${ }^{9}$ was a multicenter, randomized, double-blinded, placebo-controlled, noninferiority trial that compared ceftolozane-tazobactam plus metronidazole against meropenem in hospitalized adult patients with cIAIs. Subjects were randomly assigned to receive IV ceftolozane-tazobactam $1.5 \mathrm{~g}$ plus metronidazole $500 \mathrm{mg}$ every 8 hours or IV meropenem $1 \mathrm{~g}$ plus placebo every 8 hours for 4-14 days. In subjects with $\mathrm{CL}_{\mathrm{CR}}$ between 30 and $50 \mathrm{~mL} / \mathrm{min}$, the ceftolozane-tazobactam dose was reduced to $750 \mathrm{mg}$ every 8 hours and the meropenem dose was reduced to $1 \mathrm{~g}$ every 12 hours. Clinical outcomes were assessed at three different times during the study: the end of therapy (within 24 hours of the last dose of treatment), 
the test-of-cure (TOC) visit (24-32 days after the start of therapy), and the late follow-up visit (38-45 days after the start of therapy). The primary end point was the clinical cure at the TOC visit in the microbiological intent-to-treat (MITT) population. Secondary end points included clinical cure in the intent-to-treat (ITT), clinically evaluable (CE), and various microbiologically evaluable (ME) populations.

Of the 993 subjects who were randomized to receive ceftolozane-tazobactam plus metronidazole $(n=487)$ or meropenem $(n=506), 806(81.2 \%)$ met the criteria for the MITT population. Most of the study subjects were from Europe (76.9\%) and received therapy for up to 7 days $(-50 \%)$. The most common origin of infection was the appendix, with appendiceal perforation or abscess being the most frequent diagnosis. Infections were frequently polymicrobial, and the most common isolates included E. coli, K. pneumoniae, and $P$. aeruginosa. Clinical cure rates at the TOC visit in the MITT population were $83 \%$ with ceftolozane-tazobactam plus metronidazole and $87.3 \%$ with meropenem (weighted difference, $-4.2 \%$; two-sided $95 \%$ confidence interval [CI], $-8.91 \%$ to $0.54 \%$ ), which met the primary objective of noninferiority. Treatment failure occurred in $8.2 \%$ of patients in both groups in the MITT population at the TOC visit. Clinical failure was most commonly due to persisting or recurrent abdominal infection requiring additional intervention and the requirement for additional antibiotics for ongoing cIAIs. There were no significant differences in the clinical cure rates between groups in secondary and subgroup analyses. In patients with an ESBL-producing Enterobacteriaceae isolated, clinical cure rates were $95.8 \%$ and $88.5 \%$ in the ceftolozane-tazobactam plus metronidazole and meropenem groups, respectively, at the TOC visit. When CTX-M-14/15 ESBL-producing Enterobacteriaceae were isolated, clinical cure was observed in $13 / 13(100 \%)$ patients receiving ceftolozane-tazobactam and $8 / 11(72.7 \%)$ patients receiving meropenem.

The results of the ASPECT-cIAI trial ${ }^{9}$ were challenged by Spellberg and Brass. ${ }^{40}$ They debated that although the results met the predefined margin of noninferiority, the weighted difference and CI that only narrowly crossed zero suggests that ceftolozane-tazobactam plus metronidazole may actually be inferior to meropenem in the treatment of cIAIs. Other concerns included the difference in mortality rate $(2.3 \%$ for ceftolozane-tazobactam plus metronidazole vs $1.6 \%$ for meropenem) and the large percentage of study subjects from Eastern Europe, which may not extrapolate to patients elsewhere. The high clinical cure rates in Eastern Europe $(\sim 97 \%)$ suggest that the patient population in the ASPECT-cIAI ${ }^{9}$ trial may not be representative of patients in the USA requiring antibiotics for cIAIs. These concerns were refuted by the ASPECT-cIAI authors. ${ }^{41}$ They explained that the noninferiority margin of $-10 \%$ was predefined and supported by the FDA as the acceptable difference between the two treatment groups to be considered noninferior. This margin is actually smaller than the European noninferiority margin of $-12.5 \%$. Additionally, both ITT and per-protocol populations were assessed, which showed even smaller treatment differences. With regard to the concerns about the high clinical cure rates in Eastern Europe not representing higher-risk patients in the USA, the authors explain that this issue is present in all antimicrobial Phase III trials. Because many high-risk patients are appropriately treated empirically, they must be excluded based on the FDA standard to exclude subjects with recent antimicrobial therapy. They highlight the fact that treatment decisions are based on both clinical trials and experience in clinical practice.

ASPECT-cUTI was a multicenter, randomized, doubleblinded, placebo-controlled, noninferiority trial that compared ceftolozane-tazobactam with levofloxacin in hospitalized patients with pyelonephritis or complicated lower UTIs. ${ }^{7}$ Patients were randomly assigned to receive either IV ceftolozane-tazobactam $1.5 \mathrm{~g}$ every 8 hours or IV levofloxacin $750 \mathrm{mg}$ once daily for 7 days. Clinical and microbiological outcomes were assessed at the TOC visit (5-9 days after the last dose of the study drug) and at the late follow-up visit (21-42 days after the end of the study treatment) in the microbiological modified intent-to-treat (mMITT) and per-protocol populations. The primary end point was the composite cure, defined as achieving clinical cure and microbiological eradication of all baseline uropathogens, in the MMITT population. Secondary end points included the composite cure in the perprotocol population as well as clinical cure, microbiological cure, and composite cure in various subgroups. Superiority was defined as a positive treatment difference, with the lower bound of the $95 \%$ CI being $>0$.

In total, 1,083 patients were randomly assigned to receive ceftolozane-tazobactam $(n=543)$ or levofloxacin $(n=540)$; $73.9 \%$ met criteria for the mMITT population. Most patients (75\%) were from Europe; pyelonephritis was diagnosed in $82 \%$, and E. coli was isolated in $78.6 \%$. Other uropathogens isolated included $K$. pneumoniae, Proteus mirabilis, and $P$. aeruginosa. In the mMITT population, $26.5 \%$ of patients had a levofloxacin-resistant organism and $14.8 \%$ had ESBL-producing Enterobacteriaceae. In the mMITT population, composite cure was achieved in $76.9 \%$ and $68.4 \%$ of patients receiving ceftolozane-tazobactam and levofloxacin, respectively, at the TOC visit (difference, 8.5\%; 95\% CI, 
2.3-14.6), indicating that ceftolozane-tazobactam met the primary objective of noninferiority as well as superiority. Ceftolozane-tazobactam was also shown to be noninferior and superior to levofloxacin based on composite cure in the per-protocol population and microbiological eradication in both the mMITT and per-protocol populations. Clinical cure rates were also higher in the ceftolozane-tazobactam group in both the mMITT and per-protocol populations, meeting criteria for noninferiority but not superiority. In the mMITT population with an ESBL-producing organism isolated from baseline urine culture, composite cure at the TOC visit was achieved in 38/61 (62.3\%) and 20/57 (35.1\%) patients receiving ceftolozane-tazobactam and levofloxacin, respectively (difference, 27.2\%; 95\% CI, 9.2-42.9).

Ceftolozane-tazobactam is currently being studied at a higher dose in a multicenter, prospective, double-blinded, randomized Phase III trial (ASPECT-nosocomial pneumonia) ${ }^{42}$ for the treatment of ventilator-associated bacterial pneumonia or ventilated hospital-acquired bacterial pneumonia. The higher dose of ceftolozone-tazobactam used in this trial was justified by Xiao et $\mathrm{a}^{43}$ using Monte Carlo simulations to predict the probability of target attainment (PTA) in both the lung epithelial lining fluid (ELF) and plasma. The plasma-to-ELF penetration ratio is $\sim 50 \%$, and for the treatment of nosocomial pneumonia, doubling the approved doses for cUTIs and cIAIs is needed to achieve $>90 \%$ PTA against pathogens with an MIC up to $8 \mu \mathrm{g} / \mathrm{mL} .{ }^{43}$ In ASPECT-nosocomial pneumonia, ${ }^{42}$ patients are being randomized to receive either IV ceftolozane-tazobactam $3 \mathrm{~g}$ (comprising $2 \mathrm{~g}$ ceftolozane and $1 \mathrm{~g}$ tazobactam) every 8 hours or IV meropenem $1 \mathrm{~g}$ every 8 hours for 8 days, with 14 days of treatment for P. aeruginosa. ${ }^{42}$ The primary outcome is all-cause mortality at day 28 in the ITT population. Secondary outcomes include clinical response rates in the MITT, ITT, and CE populations at the TOC visit (7-14 days after the end of therapy), clinical response rates at the TOC visit in the subgroup of patients with $P$. aeruginosa, and all-cause mortality at day 28 in the MITT population. Enrollment in the study is estimated to be a total of 726 patients and the estimated study completion date is February 2018.

\section{Safety and tolerability}

Similar to other cephalosporins, adverse effects of ceftolozanetazobactam are generally mild in severity. In two Phase I studies assessing the PK and safety of ceftolozane-tazobactam after single and multiple doses, adverse effects were infrequent and mild. ${ }^{35,36}$ The most common adverse events included infusion site reactions such as pain or erythema (69\% of subjects receiving multiple doses) and constipation (33\% of subjects receiving single doses). ${ }^{36}$ No subjects withdrew from either study as a result of drug-related adverse effects and there were no dose-limiting toxicities observed. ${ }^{35,36}$ In a Phase II trial comparing ceftolozane-tazobactam plus metronidazole with meropenem for cIAIs, the rate of adverse events was similar between groups $(50 \%$ for ceftolozane-tazobactam plus metronidazole vs $48.8 \%$ for meropenem). ${ }^{44}$ The most common adverse events included pyrexia, nausea, vomiting, and diarrhea. Fewer adverse events in the ceftolozanetazobactam plus metronidazole group were related to the study drug than in the meropenem group (8.5\% vs $33.3 \%$, respectively). The incidence of serious adverse events was higher in patients receiving ceftolozane-tazobactam than in those receiving meropenem (17.1\% vs 5.1\%, respectively), but none of these were determined to be related to the study drug. The most common clinical laboratory abnormalities observed in both groups were elevated liver function tests.

In the ASPECT-cIAI ${ }^{9}$ trial, the incidence of adverse events was similar between treatment groups (44\% vs $42.7 \%$ for ceftolozane-tazobactam plus metronidazole and meropenem, respectively). Most adverse events were mild to moderate in severity, with the most common being nausea $(7.9 \%)$, diarrhea $(6.2 \%)$, pyrexia $(5.2 \%)$, insomnia $(3.5 \%)$, and vomiting $(3.3 \%)$ in the ceftolozane-tazobactam plus metronidazole group. The most common laboratory abnormalities were increase in liver function tests $(2.5 \%)$. Three patients $(0.6 \%)$ receiving ceftolozane-tazobactam plus metronidazole discontinued the study drug due to drugrelated adverse events compared with four patients $(0.8 \%)$ receiving meropenem. Only one drug-related serious adverse event occurred in both groups. None of the 19 patient deaths during the study were determined to be related to either drug. In the ASPECT-cUTI trial, adverse events occurred in $34.7 \%$ and $34.4 \%$ of patients in the ceftolozane-tazobactam and levofloxacin groups, respectively. ${ }^{7}$ Again, adverse events of ceftolozane-tazobactam were generally mild in severity, and the most frequent adverse events included headache $(5.8 \%)$, constipation $(3.9 \%)$, hypertension $(3.0 \%)$, nausea $(2.8 \%)$, and diarrhea (1.9\%). Two serious adverse events (both with Clostridium difficile infection) in the ceftolozane-tazobactamtreated patients $(0.4 \%)$ were attributed to the study drug. One death occurred but was not related to the study drug. As seen in other studies, the most common laboratory abnormalities were elevations in liver function tests $(1.7 \%)$, which returned to baseline in all but two cases.

Ceftolozane-tazobactam has minimal interaction with cytochrome P450 enzymes; therefore, no significant 
drug-drug interactions are expected to occur. ${ }^{26}$ No drug-drug interactions were noted in clinical trials.

\section{Patient-focused perspectives Patient adherence}

In terms of adverse events, patient adherence to ceftolozanetazobactam is expected to be similar to that for other agents used to treat cIAIs and cUTIs. As previously described, adverse events were generally mild and were similar between groups in the clinical trials, with comparable withdrawal rates. Additionally, there are currently no significant drugdrug interactions, meaning that a patient's home regimen does not need to be altered to receive the antibiotic.

Three infusions of ceftolozane-tazobactam per day are required, which is comparable to that for most other agents used for the same indications. In general, meropenem and imipenem-cilastatin are given three to four times per day, cefepime is given two to three times per day, and piperacillintazobactam is given three to four times per day. ${ }^{8}$ Levofloxacin, on the other hand, requires only one infusion per day. Additionally, when treating cIAIs, ceftolozane-tazobactam should be used in combination with metronidazole, which requires an additional three infusions per day if given in the IV mode. Carbapenems and piperacillin-tazobactam do not require the addition of metronidazole, whereas most other cephalosporins and fluoroquinolones should be combined with metronidazole. Generally, in the absence of difficulty obtaining IV access, the number of infusions per day is not an issue in hospitalized patients; however, it may be more problematic in the outpatient setting. Infusion times vary, but are similar for these medications. Ceftolozanetazobactam is to be infused over 1 hour; comparable alternatives are infused over 30-60 minutes. Additionally, similar to the carbapenems, fourth-generation cephalosporins, and piperacillin-tazobactam, there is currently no oral formulation of ceftolozane-tazobactam. In contrast, many oral formulations of fluoroquinolones are available.

Duration of therapy is also generally similar between agents. Ceftolozane-tazobactam is recommended to be given for 7 days in the treatment of cUTIs and for 4-14 days in the treatment of cIAIs. ${ }^{26}$ With the exception of levofloxacin, which has been shown to be effective when given for as short as 5 days, the duration of treatment for other antibiotics used to treat cUTIs is typically 7-14 days or longer in certain situations. ${ }^{5,7}$ The duration of therapy for cIAIs is typically 4-7 days and is generally dependent on source control rather than the antimicrobial agent used; longer durations may be necessary if adequate source control is difficult to achieve. ${ }^{8,45}$

\section{Cost considerations}

As expected of newly approved medications, ceftolozanetazobactam is costly compared to older agents used for the same indications, particularly because there are generic versions of the comparators available. The wholesale acquisition cost of ceftolozane-tazobactam is $\$ 83$ per $1.5 \mathrm{~g}$ vial or $\sim \$ 250 / \mathrm{d}$ in a patient with normal renal function. ${ }^{46}$ Several major insurance companies cover ceftolozanetazobactam as a tier 1 or 2 copayment structure, while some others have not yet made a decision but are willing to discuss on individual patient cases (personal communication, Harvard Pilgrim Health Care, BlueCross/BlueShield of MA, and Tufts Health Plan). A patient assistance program is available for some patients without insurance who meet specific criteria (accesszerbaxa.com).

\section{Availability}

In recent months, the availability of piperacillin-tazobactam and many of the carbapenems has been limited due to drug shortages. As such, many inpatient and outpatient institutions had to alter drug utilization to reflect inventory. The availability of ceftolozane-tazobactam lessens the burden of shortages in other drug classes and minimizes the potential of therapeutic interruptions.

\section{Conclusion/place in therapy}

The development of novel antibiotics is unique. Unlike chronic diseases in which lifelong therapy is indicated, infections are treated with short courses of therapy and the overuse of antibiotics leads to the development of bacterial resistance; therefore, the return on investment for antibiotics is not favorable compared to that for medications used to treat other disease states. ${ }^{47,48}$ Additionally, due to the concerns about increased resistance with overutilization of antibiotics, antimicrobial stewardship programs generally reserve novel agents for the treatment of proven MDR infections.

Ceftolozane-tazobactam is a recently approved $\beta$-lactam/ $\beta$-lactamase inhibitor for the treatment of cUTIs, and cIAIs in combination with metronidazole. Its place in therapy is currently not well established; however, it appears to have utility as a carbapenem-sparing antibiotic for the treatment of MDR Gram-negative infections. Ceftolozane's high affinity for PBP1b, PBP1c, and PBP3 and low affinity for PBP4 render it less affected by hydrolysis by AmpC $\beta$-lactamases, as well as efflux pumps and porin deficiencies; therefore, it has activity against resistant strains of $P$. aeruginosa. The addition of tazobactam gives ceftolozane activity against ESBL-producing Enterobacteriaceae. This enhanced activity 
against Gram-negative pathogens has been demonstrated in vitro and in Phase III clinical trials. The important gaps in its spectrum of activity include limited coverage against Staphylococcus spp. as well as Gram-negative anaerobes other than $B$. fragilis. It also is not active against organisms possessing metallo- $\beta$-lactamases, $K$. pneumoniae carbapenemases, or other carbapenemases. Phase III clinical trials have shown that ceftolozane-tazobactam is safe and effective in the treatment of cUTIs, and cIAIs in combination with metronidazole.

Based on the currently available data, ceftolozanetazobactam shows promise as a carbapenem-sparing definitive therapy for the treatment of infections caused by ESBLproducing Enterobacteriaceae and MDR P. aeruginosa. Although it appears that it will be used primarily as a niche antibiotic for infections caused by these resistant pathogens, ceftolozane-tazobactam in combination with metronidazole may also be appropriate for empiric therapy of health care-associated cIAIs, particularly in patients with recent antimicrobial exposure or in areas where the prevalence of MDR pathogens is high. The results of the Phase III trial for the treatment of ventilated nosocomial pneumonia, as well as clinical experience, will further define the role of ceftolozane-tazobactam in clinical practice.

\section{Disclosure}

TEB has received research funding from Actavis/Allergan and Merck and sits on a speakers' bureau for The Medicines Company. EBH has received research funding from Durata Therapeutics/Actavis, consulting honoraria from Theravance Biopharma, and sits on a speakers' bureau for The Medicines Company. MVM has received research funding from Forest Pharmaceuticals/Actavis and consulting honoraria from Cubist Pharmaceuticals. SEG declares no conflicts of interest in this work.

\section{References}

1. Sievert DM, Ricks P, Edwards JR, et al. Antimicrobial-resistant pathogens associated with healthcare-associated infections: summary of data reported to the National Healthcare Safety Network at the Centers for Disease Control and Prevention, 2009-2010. Infect Control Hosp Epidemiol. 2013;34(1):1-14.

2. Hampton T. Report reveals scope of US antibiotic resistance threat. JAMA. 2013;310(16):1661-1663.

3. Zilberberg MD, Shorr AF. Secular trends in gram-negative resistance among urinary tract infection hospitalizations in the United States, 2000-2009. Infect Control Hosp Epidemiol. 2013;34(9):940-946.

4. Babinchak T, Badal R, Hoban D, et al. Trends in susceptibility of selected gram-negative bacilli isolated from intra-abdominal infections in North America: SMART 2005-2010. Diagn Microbiol Infect Dis. 2013; 76(3):379-381.

5. Nicolle LE. A practical guide to antimicrobial management of complicated urinary tract infection. Drugs Aging. 2001;18(4):243-254.
6. Pallett A, Hand K. Complicated urinary tract infections: practical solutions for the treatment of multiresistant Gram-negative bacteria. $J$ Antimicrob Chemother. 2010;65(suppl 3):iii25-iii33.

7. Wagenlehner FM, Umeh O, Steenbergen J, Yuan G, Darouiche RO. Ceftolozane-tazobactam compared with levofloxacin in the treatment of complicated urinary-tract infections, including pyelonephritis: a randomised, double-blind, phase 3 trial (ASPECT-cUTI). Lancet. 2015; 385(9981):1949-1956.

8. Solomkin JS, Mazuski JE, Bradley JS, et al. Diagnosis and management of complicated intra-abdominal infection in adults and children: guidelines by the Surgical Infection Society and the Infectious Diseases Society of America. Clin Infect Dis. 2010;50(2):133-164.

9. Solomkin J, Hershberger E, Miller B, et al. Ceftolozane/tazobactam plus metronidazole for complicated intra-abdominal infections in an era of multidrug resistance: results from a randomized, double-blind, phase 3 trial (ASPECT-cIAI). Clin Infect Dis. 2015;60(10):1462-1471.

10. Toussaint KA, Gallagher JC. Beta-lactam/beta-lactamase inhibitor combinations: from then to now. Ann Pharmacother. 2015;49(1):86-98.

11. Bush K, Jacoby GA. Updated functional classification of beta-lactamases. Antimicrob Agents Chemother. 2010;54(3):969-976.

12. Liscio JL, Mahoney MV, Hirsch EB. Ceftolozane/tazobactam and ceftazidime/avibactam: two novel beta-lactam/beta-lactamase inhibitor combination agents for the treatment of resistant Gram-negative bacterial infections. Int J Antimicrob Agents. 2015;46(3):266-271.

13. Moya B, Zamorano L, Juan C, Perez JL, Ge Y, Oliver A. Activity of a new cephalosporin, CXA-101 (FR264205), against beta-lactamresistant Pseudomonas aeruginosa mutants selected in vitro and after antipseudomonal treatment of intensive care unit patients. Antimicrob Agents Chemother. 2010;54(3):1213-1217.

14. Livermore DM. Multiple mechanisms of antimicrobial resistance in Pseudomonas aeruginosa: our worst nightmare? Clin Infect Dis. 2002;34(5):634-640.

15. Hirsch EB, Tam VH. Impact of multidrug-resistant Pseudomonas aeruginosa infection on patient outcomes. Expert Rev Pharmacoecon Outcomes Res. 2010;10(4):441-451.

16. Kaye KS, Pogue JM. Infections caused by resistant Gram-negative bacteria: epidemiology and management. Pharmacotherapy. 2015;35(10): 949-962.

17. Nguyen HM, Shier KL, Graber CJ. Determining a clinical framework for use of cefepime and beta-lactam/beta-lactamase inhibitors in the treatment of infections caused by extended-spectrum-beta-lactamase-producing Enterobacteriaceae. J Antimicrob Chemother. 2014;69(4):871-880.

18. Park SH, Choi SM, Chang YK, et al. The efficacy of non-carbapenem antibiotics for the treatment of community-onset acute pyelonephritis due to extended-spectrum beta-lactamase-producing Escherichia coli. $J$ Antimicrob Chemother. 2014;69(10):2848-2856.

19. Rodriguez-Bano J, Navarro MD, Retamar P, Picon E, Pascual A. Beta-lactam/beta-lactam inhibitor combinations for the treatment of bacteremia due to extended-spectrum beta-lactamase-producing Escherichia coli: a post hoc analysis of prospective cohorts. Clin Infect Dis. 2012;54(2):167-174.

20. Hirsch EB, Guo B, Chang KT, et al. Assessment of antimicrobial combinations for Klebsiella pneumoniae carbapenemase-producing K. pneumoniae. J Infect Dis. 2013;207(5):786-793.

21. Hirsch EB, Tam VH. Detection and treatment options for Klebsiella pneumoniae carbapenemases (KPCs): an emerging cause of multidrug-resistant infection. J Antimicrob Chemother. 2010;65(6): 1119-1125.

22. van Duin D, Kaye KS, Neuner EA, Bonomo RA. Carbapenem-resistant Enterobacteriaceae: a review of treatment and outcomes. Diagn Microbiol Infect Dis. 2013;75(2):115-120.

23. Zasowski EJ, Rybak JM, Rybak MJ. The beta-lactams strike back: ceftazidime-avibactam. Pharmacotherapy. 2015;35(8):755-770.

24. Phe K, Johnson ML, Palmer HR, Tam VH. Validation of a model to predict the risk of nephrotoxicity in patients receiving colistin. Antimicrob Agents Chemother. 2014;58(11):6946-6948. 
25. Phe K, Lee Y, McDaneld PM, et al. In vitro assessment and multicenter cohort study of comparative nephrotoxicity rates associated with colistimethate versus polymyxin B therapy. Antimicrob Agents Chemother. 2014;58(5):2740-2746.

26. Merck \& Co., Inc. [webpage on the Internet]. Zerbaxa Prescribing Information. Available from: http://www.zerbaxa.com/pdf/ PrescribingInformation.pdf. Accessed February 26, 2016.

27. Cho JC, Fiorenza MA, Estrada SJ. Ceftolozane/tazobactam: a novel cephalosporin/beta-lactamase inhibitor combination. Pharmacotherapy. 2015;35(7):701-715.

28. Bulik CC, Tessier PR, Keel RA, Sutherland CA, Nicolau DP. In vivo comparison of CXA-101 (FR264205) with and without tazobactam versus piperacillin-tazobactam using human simulated exposures against phenotypically diverse gram-negative organisms. Antimicrob Agents Chemother. 2012;56(1):544-549.

29. Craig WA, Andes DR. In vivo activities of ceftolozane, a new cephalosporin, with and without tazobactam against $P$ seudomonas aeruginosa and Enterobacteriaceae, including strains with extended-spectrum beta-lactamases, in the thighs of neutropenic mice. Antimicrob Agents Chemother. 2013;57(4):1577-1582.

30. Moya B, Zamorano L, Juan C, Ge Y, Oliver A. Affinity of the new cephalosporin CXA-101 to penicillin-binding proteins of Pseudomonas aeruginosa. Antimicrob Agents Chemother. 2010;54(9):3933-3937.

31. Sader HS, Rhomberg PR, Farrell DJ, Jones RN. Antimicrobial activity of CXA-101, a novel cephalosporin tested in combination with tazobactam against Enterobacteriaceae, Pseudomonas aeruginosa, and Bacteroides fragilis strains having various resistance phenotypes. Antimicrob Agents Chemother. 2011;55(5):2390-2394.

32. Clinical and Laboratory Standards Institute. Performance Standards for Antimicrobial Susceptibility Testing; Twenty-Fifth Informational Supplement. M100-S25. Wayne, PA: Clinical and Laboratory Standards Institute; 2015.

33. Farrell DJ, Flamm RK, Sader HS, Jones RN. Antimicrobial activity of ceftolozane-tazobactam tested against Enterobacteriaceae and Pseudomonas aeruginosa with various resistance patterns isolated in U.S. Hospitals (2011-2012). Antimicrob Agents Chemother. 2013; 57(12):6305-6310.

34. Snydman DR, McDermott LA, Jacobus NV. Activity of ceftolozanetazobactam against a broad spectrum of recent clinical anaerobic isolates. Antimicrob Agents Chemother. 2014;58(2):1218-1223.

35. Ge Y, Whitehouse MJ, Friedland I, Talbot GH. Pharmacokinetics and safety of CXA-101, a new antipseudomonal cephalosporin, in healthy adult male and female subjects receiving single- and multiple-dose intravenous infusions. Antimicrob Agents Chemother. 2010;54(8): 3427-3431.

36. Miller B, Hershberger E, Benziger D, Trinh M, Friedland I. Pharmacokinetics and safety of intravenous ceftolozane-tazobactam in healthy adult subjects following single and multiple ascending doses. Antimicrob Agents Chemother. 2012;56(6):3086-3091.
37. Wooley M, Miller B, Krishna G, Hershberger E, Chandorkar G. Impact of renal function on the pharmacokinetics and safety of ceftolozanetazobactam. Antimicrob Agents Chemother. 2014;58(4):2249-2255.

38. MacGowan AP, Noel AR, Tomaselli SG, Nicholls D, Bowker KE. Pharmacodynamics of ceftolozane plus tazobactam studied in an in vitro pharmacokinetic model of infection. Antimicrob Agents Chemother. 2015;60(1):515-521.

39. FDA [webpage on the Internet]. FDA Drug Safety Communication: FDA Cautions about Dose Confusion and Medication Errors for Antibacterial Drug Zerbaxa (Ceftolozane and Tazobactam). Available from: http://www.fda.gov/Drugs/DrugSafety/ucm445919.htm. Accessed January 7, 2016.

40. Spellberg B, Brass EP. Noninferiority doesn't mean not inferior. Clin Infect Dis. 2016;62(4):525-526.

41. Solomkin JS, Hershberger E, Eckmann C. Response to Spellberg and Brass. Clin Infect Dis. 2016;62(4):526.

42. ClinicalTrials.gov [webpage on the Internet]. Safety and Efficacy Study of Ceftolozane/Tazobactam to Treat Ventilated Nosocomial Pneumonia (ASPECT-NP). Identifier NCT02070757; 2015. Available from: https:// clinicaltrials.gov/ct2/show/NCT02070757?term=ceftolozane \&rank=2. Accessed January 7, 2016.

43. Xiao AJ, Miller BW, Huntington JA, Nicolau DP. Ceftolozane/ tazobactam pharmacokinetic/pharmacodynamic-derived dose justification for phase 3 studies in patients with nosocomial pneumonia. J Clin Pharmacol. 2016;56(1):56-66.

44. Lucasti C, Hershberger E, Miller B, et al. Multicenter, double-blind, randomized, phase II trial to assess the safety and efficacy of ceftolozanetazobactam plus metronidazole compared with meropenem in adult patients with complicated intra-abdominal infections. Antimicrob Agents Chemother. 2014;58(9):5350-5357.

45. Sawyer RG, Claridge JA, Nathens AB, et al. Trial of short-course antimicrobial therapy for intraabdominal infection. $N$ Engl J Med. 2015; 372(21):1996-2005.

46. Cluck D, Lewis P, Stayer B, Spivey J, Moorman J. Ceftolozanetazobactam: a new-generation cephalosporin. Am J Health Syst Pharm. 2015;72(24):2135-2146.

47. Boucher HW, Talbot GH, Benjamin DK Jr, et al. 10 x '20 progress development of new drugs active against gram-negative bacilli: an update from the Infectious Diseases Society of America. Clin Infect Dis. 2013;56(12):1685-1694.

48. Boucher HW, Talbot GH, Bradley JS, et al. Bad bugs, no drugs: no ESKAPE! An update from the Infectious Diseases Society of America. Clin Infect Dis. 2009;48(1):1-12.
Therapeutics and Clinical Risk Management

\section{Publish your work in this journal}

Therapeutics and Clinical Risk Management is an international, peerreviewed journal of clinical therapeutics and risk management, focusing on concise rapid reporting of clinical studies in all therapeutic areas outcomes, safety, and programs for the effective, safe, and sustained use of medicines. This journal is indexed on PubMed Central, CAS,

\section{Dovepress}

EMBase, Scopus and the Elsevier Bibliographic databases. The manuscript management system is completely online and includes a very quick and fair peer-review system, which is all easy to use. Visit http://www.dovepress.com/testimonials.php to read real quotes from published authors. 